\title{
A Theoretical Study of Synaesthesiaby Music Sense Organs and Creative Thinking
}

\author{
Wen-Si OU-YANG \\ Music College, Shaanxi Normal University, No. 620, West Chang' an Avenue, Chang' an District, \\ Xi'an 710119, Shaanxi, China \\ ouyangjusco@snnu.edu.cn
}

Keywords: Music, Visual Sensation, Auditory Sensation, Olfactory Sensation, Creative Thinking.

\begin{abstract}
Music appreciation has always been a popular topic as a complex-yet-unique psychological experience and approach, which can improve personal music aesthetics, provoke diverse perceptions, develop intuition and imagination, and help achieve cultural accomplishment. In order to enhance creative thinking ability, this paper proposes to cultivate music appreciation more efficiently through sufficient visual sensation (sight), auditory sensation (sound) and olfactory sensation (smell).
\end{abstract}

\section{Introduction}

Creative thinking refers to the mind processes involved in the conception of new and innovative ideas. It is a high-level psychological activity which elicits originality and diversity. Music experience is a developmental and creative thinking process that effectively relies on various music languages and messages [1]. Musicians capable of deciphering these languages and messages can often produce distinct possibilities, and this is what the term music thinking entails. Comparison between music thinking and music experience reveals the notable difference between the two terms. Music experience is a sensation represented by certain properties and characteristics of the music, for example the style of the music or the tone of the instrument. However music thinking relates to the essence of the music and music-related matters and the correlative patterns between them. The process of experiencing music in a creative way is the in-depth realization and embodiment of music thinking, for the purpose of understanding of various musical objects from a novel standpoint, and being able to blend 'novelty' with 'beauty,' and achieving brand-new creative results. Fusing motion and tranquility, whilst combining the enjoyment of music with images and smell, provides a new insight that people pursue into music appreciation and understanding.

In "A Glossary of Literary Terms," the American literary critic, Meyer Howard Abrams (1912-2015) defined synaesthesia as follows. "Synaesthesia [2] is the psychological term for experiencing two or more modes of sensation when only one sense is being stimulated. In literature the term is applied to descriptions of one kind of sensation in terms of another; color is attributed to sounds, odor to colors, sound to odors, and so on." Hence, synaesthesia is a regular form of psychological activity involving the processes of comparison, analysis and association. Qian Zhongshu provided an apt metaphor for synaesthesia: "the daily experience [3] shows that the visible sensation, auditory sensation, touch sensation, olfactory sensation and gustatory sensation can be interacted with each other. The boundary between the eyes, ears, noses and tongues can be blurred. As a result, the color seems to have a temperature, the sound seems to have an imagination, and the cold and hot sensation seems to have a weight, the smell seems to have a tangible blade." This description suggests that 'experience' and 'association' are both prerequisites of synaesthesia. The frequent activities of recognition, comparison and classification in daily life all depend on existing physiological and social experience. At the same time, our ability for logical thinking, analysis and comparison decisively influences one's psychological feelings., the correlation and conversion of experiences in auditory, visual and other sensations exerts a psychological association, thereby generating synaesthesia (also known as transference and sensory affinity).

The famous German composer, Robert Schumann (1810-1856) had a profound insight into this 
phenomenon.

Can we deny the achievements of nature and the use of wondrous beauty into music? Italy, the Alps, magnificent ocean views, spring, all the fantastic scenery around us, are all things depicted to us by music. Even inconsequential and non-generic phenomena are able to endow music with a kind of changeable and specific visual property. Music should astound us by its ability to represent so many things. A composer once said that when he wrote a piece of music, he would imagine a butterfly lying on a leaf floating along the stream. This imagination gave his composition a subtle flavor of extreme softness mixed with childhood fantasy and romance, thus producing a vivid impression of this scene among the audience [4].

This passage from Schumann implies a psychological tendency of the human minds to sympathize with objective things. Composers convert their direct experience of the world and their emotions into music thinking, and convey their feelings in the musical notes, arousing people via a synaesthesia of auditory sensation. When the sounds meet their ears, people form an associative picture in their mind, and tune in to the relevant artistic connotations which composers want to convey. This form of synaesthesia creates an important bridge between musical language and visual language.

\section{Basic Characteristics of Music Appreciation}

Sound is generated by a series of vibrations, which in turn are transmitted via the medium of air to the eardrum and create an auditory image to be subsequently judged and analyzed by the brain. The aesthesia and auditory sensation form the foundation and development pattern for human consciousness, mentality and cognition. Hence, music appreciation is a complex amalgamation of musical sensation and aesthetic feelings, resulting in a unique sense of control, experience and memory, which are allotted to a specific association space in the brain.

\section{Sense of Control}

The objective world plays a direct role on the subjective thinking of mankind. A sensation is relied on to provide us with an explanation for all objective things in life, while perception is a comprehensive reflection of objective elements based on a variety of sensations. During the process of listening to music in order to elicit perception in the brain, the audience will initially receive an superficial sensation of the sound of the music, based on pitch, rhythm, tone quality and tempo. However, the sensation does not stop at the level of direct sensation. Instead, it converts into a form of sensual thinking in the brain, where the basic elements of the sound (e.g. the pitch) will be subsequently analyzed and judged. Thereupon the sensation is upgraded to the perception of the music's theme, style, form, feeling and philosophical ideology. Ultimately, the emotional experience of the music and the image associated with it can be expressed. One's perception of music inevitably varies with the person's individual accumulation of knowledge and experience.

\section{Experience and Memory}

When we perceive music through an auditory sensation, the brain will actively process these auditory messages, with the pre-existing memory and experience affecting this process in a penetrative fashion. For example, the theme of Beethoven's Symphony No. 5 in C minor was supposedly formed to represent 'Fate knocking on the door'. Therefore, whenever someone hears three repetitions of a certain tone followed by a different tone, three diatonic degrees below, as in Beethoven's Fourth Piano Concerto or Appassionata Sonata, listeners associate the sound with the knock of Fate.

Hence, people tend to form some memories and experiences based on a specific object or sound with which they have previously come into contact. These memories and experiences are usually activated by the stimulations of certain factors or implicit relationship. People can improve their sensation ability by accumulating and comparing their experiences. The accumulation of experience, 
knowledge and memory then elicits an inclination in the brain to form a relatively fixed image and cognition process for specific sensations and phenomena. It is interesting to note that, even if auditory conditions change, the impression stored in the brain over a long duration does not significantly alter. Consider the color pink which is often associated with something feminine. Even when pink is associated with a male, our perception of the pink color remains the same. In the same way, an experience or memory, provide a source of reference for us when observing, participating, and analyzing the music to which we are listening. The experience and memory thus lay an important foundation for the development of our consciousness and for making sense of our ambiguous world.

\section{Association Space}

When music is first transmitted, biological signals are primarily received through nerves in the eardrum, subjected to numerous code conversions in the auditory nerves, and then processed by functional areas in the brain before we finally gain perception and cognition of the sensation, forming a spiritual world enormously different from the objective world, although sharing a close relationship with nature and society.

In the process of music appreciation, the descriptive nature of the music can resonate from its listeners, who freely associate the music with their life and emotional experience. The construction and experience of the imagination space irrefutably has various social and cultural factors. It represents not only the physiological reactions which occur in the cerebral cortex, but also the social and cultural experience of individuals. Music is therefore often associated with one's own experience, images stored in our memory, based on plots and characters from dramas and literature, also philosophical ideas. In this way music is associated with the reality world, and enriches the extent of psychological space transfer between itself and other information.

Musical experience is a combination of human sensibility and rationality. Musical sensation is then followed by a response of human feeling and meditation to perceived meanings. Making sense of the ideas evoked by the music, based on an accumulation of experience and memories, yields

distinct association space. Consider 《Peter and the Wolf》 (op.67 1936) by Sergei-Sergeyevich·Prokofiev (1891-1953), whose most valuable feature is a close link between the music and the plot as a perfect combination of the conflicts depicted in the drama and its expression of feelings. The music, which has a very logical and systematic structure, perfectly corresponds with the development of the story plot and narration of the aside. Owing to the spectacular artistic charms and lifelike characters, the audience feels as if they are literally present within the scene. This classical piece illustrates to children, in the form of music, that instead of being gripped with fear, we can defeat the most formidable of enemies by remaining calm and sticking together. Another example is Zigeunerweisen (Op.20 1878) by Pablo de Sarasate (1844-1908). This musical composition depicts the legendary and free life of the Gypsy. Based on the titles and non-music information about the work's background, and the emotions that are expressed in the three parts of the piece, audience can generate the association of these specific emotional contents: the poor life led by roaming gypsies which endured for many generations, could not force this group of society from living an unhurried, carefree and optimistic life whilst continuing to love their music.

Regardless of the type of music concerned, it is not until after the music is sensed that the listener gains a perception such as we have described above, based on one's understanding of the life and characters in the respective work, and under the guidance of one's rational thoughts. For people who have not heard of this particular work, on the other hand, an understanding would be limited to some written descriptions. Hence, intuitively sensing and experiencing the music is the essence of music appreciation. This situation highlights the shortcomings of the traditional pattern of music appreciation. For a given work that is to be sensed only through auditory sensation, a perception and association of this type of work will vary with the experience and knowledge background of individuals. An in-depth understanding of this work will not be achieved without referring to relevant information. Our sensory organs are independent and limited in their own ways. In this 
context, an understanding of the music's theme can be facilitated by combining the sound of the music with an image, with music content as primary medium, yet enabling the abstract music to be visualized. This provides a completely new means of music enjoyments and has unrivaled advantages over traditional music appreciation.

Consider the French composer and first exponent of musical impressionism, Debussy. While listening to his music, the audience will automatically visualize one image after another in their minds. These clear images are formed from a combination of impressionism, poetry and profound mysticism. Compared to lingual and auditory sensations, the intuitive visual sensation is more capable of interpreting the profound connotations synonymous with Debussy's music. Debussy once expressed his objection to the label of 'impressionism', alternatively suggesting that what he pursued was just the "introduction of something new into the music" and the "creation of a unique reality in the music". He strove to add "taste" to the music and explore the unique truth via the olfactory sensation. We then find that the music is no longer abstract; the music is embodied by images, smell and taste. Therefore, only when the sensory organs of the entire body are used (e.g. visual sensation, auditory sensation, touch sensation and olfactory sensation) can we understand Debussy; and when the essence of Debussy's music is comprehended, an improvement in creative thinking, enriched associations and an increase in perceptual feelings results. Another example is Danseuses de Delphes - Préludes Book I 1910 from Debussy. This piece of music used extremely colorful chords to impress listeners across their visual,, auditory, tactile and olfactory sensations. It depicts a vast space and soft wind experienced in the evening, the colors present at dusk together with the various scents and sounds, creating an intoxicating countryside atmosphere.

Visual sensation, auditory sensation, tactile sensation and olfactory sensation, are all important psychological processes necessary in music appreciation. Human thought processes are continuously advancing and innovating. We can no longer merely sense all elements of music through our visual and auditory sensations. Conversely, we should keep accumulating our experiences and encourage people's creative thinking in various ways to broaden the scope of perceptions and associations. Moreover, owing to a rapid technological development and an increased interaction of music with other fields, we are consequently seeing a vast increase in music appreciation with practical uses. The auditory sensation is no longer the only way to sense music. Visual sensation, tactile sensation and olfactory sensations enable people to perceive their world from more diverse perspectives and incorporating a variety of thinking modes. In addition, this broadens people's multi-dimensional aesthetic space, stimulates creativity and inspiration, and ultimately enhances the charm and beauty of the art of music.

\section{References}

[1] Cihai Editing Committee. Chihai(An Encyclopedia of the Chinese Language). Shanghai: Shanghai Lexicographic Publishing House , 1989: 208

[2] M.H. Abrams. Dictionary of Literary Terms. Translated by Wu Songjiang et al. Beijing: Beijing University Press, 2009: 630.

[3] Qian Zhongshu. Four Collections of Old Stories Shanghai: Shanghai Classics Publishing House, 1989: 52.

[4] Robert Schuman. Discourse on Music and Musicians. Translated by Chen Dengyi et al. [M]. Beijing: People's Music Publishing House, 1962: 82

[5] Leonard B. Meyer and Grosvenor W. Cooper, "The Rhythmic Stucture of Music", The University of Chicago Press, 1960

[6] Barbara R.Barry,“Musical Time:The Sense of Order”, Pendragon Press Stuyvesant, NY1990.

[7] Karlheinz Stockhausen,“Structure and Experiential Time, ”Die Reihe 1-4,1958-60.

[8] Tom Kelley, David Kelley, Creative Confidence:Unleashing the Creayive Potential Within Us 
All, 9/15/13 Crown Business, Massachusetts, 2013

[9] Tomsa Vogel, Breakthrough Thinking: A Guide to Creative Thinking and Idea Generation, HOW Books,Florida, 2014

[10] Victor Zuckerkandl, The sense of Music, Princeton University Press, New Jersey, 1970 